\title{
Non-Recurrent Laryngeal Nerve and Concurrent Vascular Variants: A Review
}

\author{
Emmanouil Bakalinis ${ }^{1,2}$, Ioannis Makris², Theano Demesticha ${ }^{2}$, Georgios Tsakotos², \\ Panagiotis Skandalakis ${ }^{2}$, Dimitrios Filippou ${ }^{2}$
}

\author{
${ }^{1}$ Second Department of Surgery, Metaxa \\ Cancer Hospital, Piraeus, Greece, \\ ${ }^{2}$ Department of Anatomy and Surgical \\ Anatomy, Medical School, National and \\ Kapodistrian University of Athens, Athens, \\ Greece.
}
Correspondence: mbakalinis@gmail.com
Tel.: + 306984852577
Fax.: + 302109760033

Received: 8 July 2018

Accepted: 28 November 2018

Key words: Thyroidectomy - Recurrent Laryngeal Nerve - Variant - Aberrant Right Subclavian Artery.

\begin{abstract}
Objective. The purpose of this paper is to review the current data on the coexistence of non-recurrent laryngeal nerve (RLN) and vascular variations. Methods. A systematic literature search was conducted on MEDLINE for case reports, original articles and reviews regarding the presence of non-RLN and coexisting vascular variants. Results. From the literature search, 104 cases of non-RLN with confirmed vascular variants were reported. More specifically, 97.7\% ( $n=101)$ of cases involved a right and $2.3 \%(n=3)$ a left non-RLN. The most common concurrent vascular variant reported with a right non-RLN was an aberrant right subclavian artery $(97 \% ; n=98)$. One case report $(0.9 \%)$ of an intrathyroidal right common carotid artery was noted and 2 cases (1.9\%) were associated with normal vascular anatomy. Furthermore, all 3 cases of a left non-RLN were associated with a right aortic arch, while 2 of them were also accompanied with situs inversus. Conclusions. The presence of vascular variations of the great vessels must prompt the surgeon to search for a non-RLN. Intraoperative neuromonitoring increases the detection rate of non-RLN. Further research is required to determine anatomic landmarks for the perioperative identification of a non-RLN, allowing its protection from potential injury.
\end{abstract}

\section{Introduction}

Surgery is indicated for the treatment of both benign and malignant thyroid diseases. Thyroidectomy is a common procedure, as reflected in the 72,344 total thyroidectomies performed in the United States in 2011 (1). In the late $19^{\text {th }}$ century, the work of Kocher revolutionized the surgical management of thyroid disease, thus converting an operation with unacceptably high morbidity to a safe procedure (2). A bloodless field and thorough understanding of the regional normal and variant anatomy are crucial for an efficient and safe operation.

The recurrent laryngeal nerve (RLN) originating from the cervical vagus (cranial nerve $\mathrm{X}$ ) provides sensory and motor function to the larynx. Sensory function involves the subglottic region while all the laryngeal muscles, except for the cricothyroid are innervated by the RLN. This nerve is responsible for both vocal cord abduction and adduction.

Damage to the RLN during thyroidectomy is responsible for vocal cord paralysis. On the right, the RLN courses posterior to 
the right subclavian artery and ascends superiorly to the lateral trachea located in the tracheoesophageal groove before entering the larynx at the level of the first tracheal ring. On the left, the RLN courses posteriorly to the aortic arch at the level of the ligamentum arteriosum, traveling along the tracheoesophageal groove superiorly before entering the larynx. Non-recurrent laryngeal nerve (non-RLN) is a rare anatomic variant with a reported incidence of $0.3-0.8 \%$ on the right and $0.004 \%$ on the left side (3). In that case, the nerve enters the larynx directly after its origin from the cervical vagus. The risk of injury to the RLN is $1-2 \%$. On the other hand, the risk of injury to a non-RLN skyrockets to $12.9 \%$, with the first report of such an injury in 1932 given by Pemberton $(4,5)$. Furthermore, non-RLN has been observed with concurrent variants of the great vessels. The presence of an aberrant right subclavian artery (ARSA) or arteria lusoria originating from the aortic arch is associated with the presence of a right non-RLN. The purpose of this study is to review current evidence on the coexistence of vascular variants and non-RLN to raise surgeons' awareness, thus preventing potential injury to the nerve.

\section{Materials and Methods}

A systematic search of international literature indexed in MEDLINE was conducted. In June 2018, the PubMed search engine was accessed for original articles, cadaveric reports, case reports and reviews regarding non-RLN and confirmed concurrent vascular variations. The search terms thyroid, thyroidectomy, non-recurrent laryngeal nerve,

Table 1. The Number of Non-RIns Reported in the International Literature, Grouped as Right and Left Side Nonrlns and their Associated Vascular Variant

\begin{tabular}{|c|c|c|c|c|}
\hline Study & $\begin{array}{l}\text { Non-RLN } \\
\text { cases }(n)\end{array}$ & $\begin{array}{l}\text { Right non- } \\
\text { RLNs (n) }\end{array}$ & $\begin{array}{l}\text { Left non- } \\
\text { RLNs (n) }\end{array}$ & Associated vascular variant \\
\hline Mahmodlou et al. (3) & 1 & 1 & - & ARSA \\
\hline Toniato et al. (4) & 31 & 31 & - & ARSA (only 5 cases verified) \\
\hline Morais et al. (9) & 2 & 2 & - & ARSA+ common trunk of common carotids \\
\hline Hong et al. (11) & 15 & 15 & - & ARSA \\
\hline Natsis et al. (12) & 6 & 6 & - & ARSA \\
\hline Baker et al. (13) & 1 & 1 & - & Intrathyroidal CCA \\
\hline Obaid et al. (14) & 1 & $1^{*}$ & - & Normal anatomy \\
\hline Sagayaraj et al. (15) & 3 & 3 & - & ARSA \\
\hline Masuoka et al. (16) & 1 & - & 1 & Right-sided aortic arch + Aberrant left SCA \\
\hline Niu et al. (18) & 26 & 26 & - & ARSA \\
\hline Tateda et al. (19) & 1 & 1 & - & Normal anatomy \\
\hline Henry et al. (20) & 33 & 31 & 2 & $\begin{array}{l}\text { ARSA (right non-RLN), Right aortic arch + situs } \\
\text { inversus (left non-RLN) }\end{array}$ \\
\hline Marchesi et al. (21) & 7 & 7 & - & ARSA \\
\hline Patron et al. (22) & 1 & 1 & - & ARSA \\
\hline lorgulescu et al. (23) & 1 & 1 & - & ARSA \\
\hline Total & 104 & $101(97.1 \%)$ & $3(2.9 \%)$ & $\begin{array}{l}\text { Right non-RLN:ARSA }(97 \%, n=98) \text {; Normal anatomy } \\
(1.9 \%, n=2) \text {; Intrathyroidal CCA }(0.9 \%, n=1) \text {; Left non- } \\
\text { RLN: Right aortic arch }(100 \%, n=3)\end{array}$ \\
\hline
\end{tabular}

*List of abbreviations: non-RLN= right non-recurrent laryngeal nerve; ARSA= aberrant right subclavian artery; $C C A=$ common carotid artery SCA = subclavian artery 
vascular variants, vascular variations and aberrant right subclavian artery were used. No restrictions were taken regarding the year the articles were published, owing to the rarity of the non-RLN. Cases of non-RLN were recorded and analyzed for concurrent vascular variants. In the event of confirmed coexisting vascular variations, cases were further grouped as right and left non-RLNs, and the incidence of specific vascular variants was calculated using Microsoft Excel version 2016 MSO (16.0.9330.2124) 32-Bit (Microsoft Corporation).

\section{Results}

From the literature search, 15 articles met the criteria to be included in our review. Ex- traction of data from these studies identified 104 cases of non-RLN in living and cadaveric specimens with confirmed concurrent vascular variations. Statistical analysis of the data (summary of the results shown in Table 1) revealed the following: $97.1 \%(n=101)$ cases regarded a right, while $2.3 \%(n=3)$ a left non-RLN. What is more, the majority of right non-RLN were accompanied with an ARSA in $97 \%$ of cases $(n=98)$. One case of right non-RLN involved an intrathyroidal right common carotid artery (CCA) $(0.9 \%)$, while in 2 cases, normal vascular anatomy was reported (1.9\%). Furthermore, all left non-RLNs were associated with a right aortic arch, while two of them were also related with situs inversus (Figure 1).
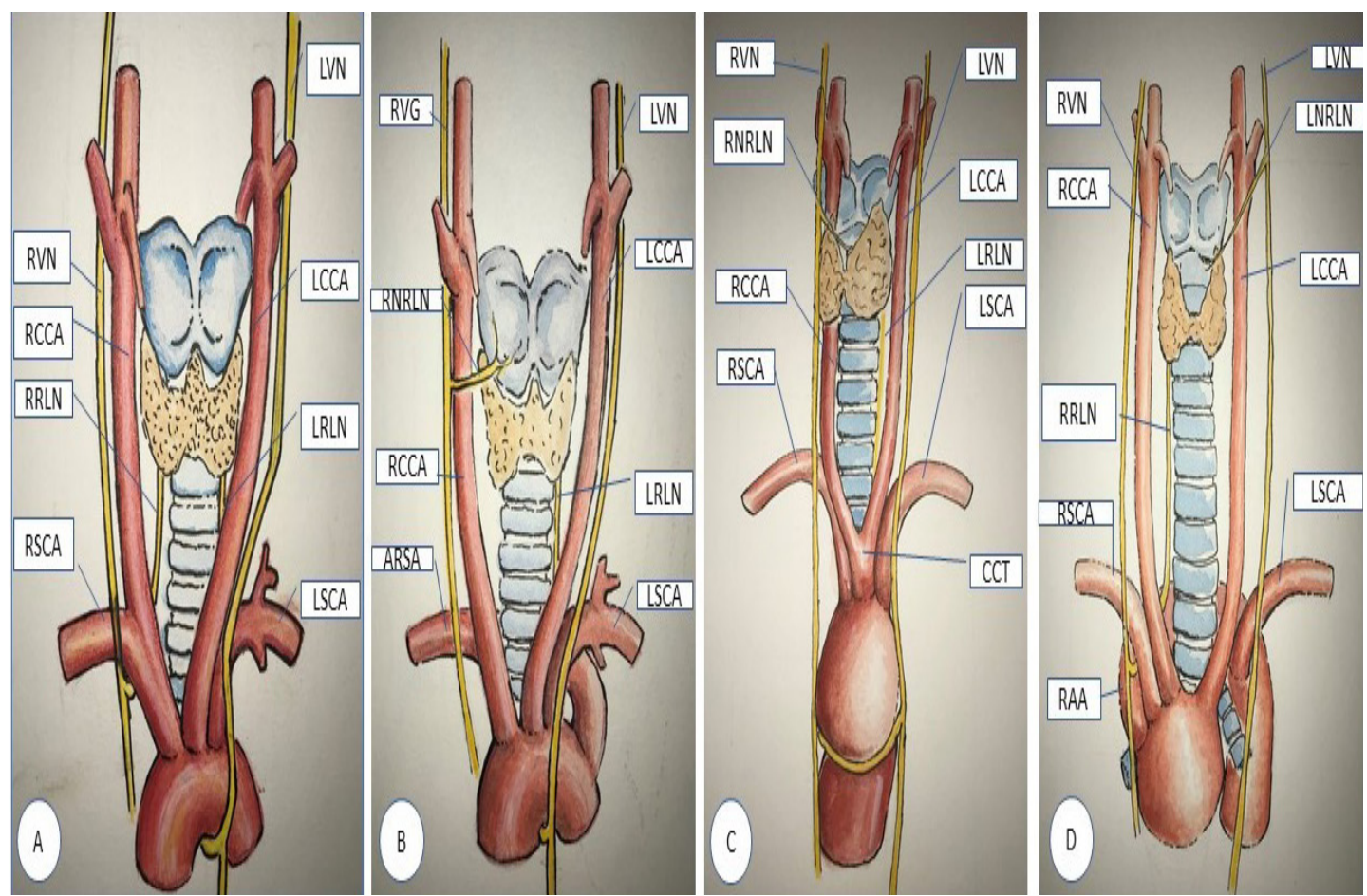

Figure 1. Schematic presentation of the recurrent laryngeal nerve $(R L N)$ and vessel anatomy. $A=$ Normal anatomy; B=Right non-RLN and ARSA; C=Right non-RLN and Intrathyroidal right common carotid artery [Adapted from Baker et al. (12)]; $D=L e f t$ non-RLN and Right-sided aortic arch; RVN=right vagus nerve; LVN=Left vagus nerve; RRLN=Right recurrent laryngeal nerve; $L R L N=$ Left recurrent laryngeal nerve; $R C C A=$ Right common carotid artery; LCCA=Left common carotid artery; RSCA=Right subclavian artery; LSCA=Left subclavian artery; RNRLN=Right non recurrent laryngeal nerve; ARSA=Aberrant right subclavian artery; LNRLN=Left non recurrent laryngeal nerve; $\mathrm{CCT}=$ Common carotid trunk. 


\section{Discussion}

Prevention of the dreaded complication of vocal cord paralysis from RLN damage during thyroid gland surgery requires an indepth knowledge of the regional anatomy and its variants. Non-RLN is a rare variant, with a reported incidence of $0.3-0.8 \%$ on the right and $0.004 \%$ on the left side, and was first described in 1823 by G.W Stedman (3, 6). Data from studies evaluating the intraoperative use of neuromonitoring for prevention of RLN injury suggest a higher incidence of non-RLN,, as high as 6\% (7). The risk of injury to the RLN is $1-2 \%$, while in contrast the risk of injury to a non-RLN skyrockets to $12.9 \%$, with the first report of such an injury in 1932 given by Pemberton $(4,5)$.

A brief review of thyroid and regional vasculature embryology may help elucidate the development of such anatomic variations. The arteries of the head and neck derive from six pairs of aortic arches, formed by the fifth gestational week. Each vessel follows a course along the axis of the pharyngeal arches and is accompanied by a cranial nerve. The third aortic arch forms the common and part of the internal carotid artery, while the fourth arch forms the aortic arch to the left and the proximal part of the subclavian artery to the right. The RLN, a branch of the vagus nerve (cranial nerve $\mathrm{X}$ ), derives from the sixth pharyngeal arch and accompanies the sixth aortic arch. Because the right sixth aortic arch degenerates during fetal development, the right RLN is translocated superiorly, coursing around the right subclavian vein. In contrast, the left sixth aortic arch remains, hence the left RLN pivots around the ductus arteriosus, which in the neonate forms the ligamentum arteriosus of the aortic arch (8). From these data, we can extrapolate possible mechanisms responsible for the presence of a non-RLN. Anomalous regression of the distal dorsal aorta leads to the aberrant origin of the right subclavian artery from the aortic arch. As a result, the right RLN fails to loop around the right subclavian artery and becomes a right non-RLN. To the left, for a left non-RLN to occur, concurrent regression of the fourth and sixth aortic arches is required.

Observation of the course of the nonRLN allows its classification into two types: Type I and II $(9,10)$. Type I non-RLN arises directly from the cervical vagus and travels along the superior thyroid pedicle, while type II travels along the inferior thyroid artery. Hong $\mathrm{KH}$ et al. proposed an alternative, more detailed classification based on their observations on 15 patients with right non-RLN. They classify them into four categories: descending, vertical, ascending and V-shaped, with an incidence of 33\%, 27\%, $20 \%$ and $20 \%$ accordingly. This group of patients consisted of 13 females and two males who were found to have a retroesophageal origin of the subclavian artery. The authors also noted a variable location of the inferior thyroid artery but could not compare its relationship to the non-RLN (11).

Identifying a non-RLN is almost always associated with variants of the great vessels. The most common vascular variant associated with a non-RLN is the ARSA, arising from the aortic arch, following a retroesophageal course. This subclavian artery variant, also known as arteria lusoria, may present with dysphagia in $5 \%$ of cases. A study of 237 Greek cadavers by Natsis et al. demonstrated six cases of ARSA (2.2\%). In 2 cases the ARSA was also accompanied by a common trunk of the left and right common carotid arteries. A female predominance was noted, with a 2:1 ratio. All ARSAs were accompanied with a right non-RLN (12). In the study by Toniato et al. 31 cases of a right non-RLN were reported in a series of 6000 patients who underwent surgery for thyroid disease. However, not all reported cases were included in our review because only 5 of them had a confirmed concurrent vascular variant (4). 
In our review, there were cases where a right non-RLN was accompanied by extremely rare vascular anatomic variants. In a case report by Baker et al. an intrathyroidal right common carotid artery with a nonRLN was described in a 23-year-old patient. Although medial displacement of the common carotid artery has been observed in other studies, this report is the first to describe such an aberrant, intrathyroidal course of the carotid artery (13). In contrast, Obaid et al. identified the extremely rare coexistence of a right non-RLN and a right RLN without any associated vascular variant, during a combined parathyroidectomy and thyroidectomy (14). Sagayaraj et al. propose a medially placed vagus in relation to the common carotid artery as an operative marker of a non-RLN. Their conclusions were based on the intraoperative findings of three patients, who were shown postoperatively to have aberrant subclavian artery anatomy (15).

The left non-RLN comprises an extremely rare variant, as has already been stated. When present, it is usually associated with situs inversus. Masuoka et al. attempted to clarify the etiology of left non-RLN. Their initial hypothesis, that the coexistence of a right-sided aortic arch and an aberrant left subclavian artery indicates a left non-RLN, was invalidated from their observations of 4 patients. From that sample, only one patient validated the initial hypothesis, thus demonstrating a left non-RLN. By examining the results of Magnetic Resonance Angiography of the aforementioned patients, the researchers recognized the presence of a descending aorta diverticulum at the point of origin of the aberrant subclavian artery in the three patients with RLNs. In contrast, the diverticulum was absent in the patient with the left non-RLN and the descending aorta traveled straight down after the aortic arch. The authors concluded that the combination of a right-sided aortic arch, an aber- rant left subclavian artery and the absence of an aortic diverticulum suggests a left nonRLN (16).

Prevention of injury to a non-RLN is of utmost importance. As has been demonstrated in our review, non-RLN is almost always associated with vascular variations. Therefore, efforts should be focused on the preoperative identification of such variants. A preoperative neck and chest CT or MRI scan can accurately demonstrate vessel anatomy. However, this practice raises the question of increased healthcare costs for the identification of a rare anatomic variation and high radiation dosage in the case of a CT scan. Considering this, our proposal is the neck CT or MRI scan performed for thyroid cancer or large goiters. Another less expensive and more readily available approach is the use of ultrasound. This also has the advantage of no radiation. On the other hand, it is operator dependent.

Intraoperatively, careful dissection is mandatory. When the surgeon fails to identify the recurrent inferior laryngeal nerve in its predicted place, he should consider the presence of a non-RLN. Intraoperative neuromonitoring helps the surgeon to understand the anatomy and function of the nerve structures better and it does not add significantly to the cost of the operation. Donatini et al. identified and preserved a right non-RLN in 11 cases, in a series of 402 thyroidectomies utilizing constant neuromonitoring. In the absence of neuromonitoring devices, the surgeon should dissect the vagus nerve and recognize the non-RLN at its origin from the cervical vagus, and follow its course to the cricothyroid muscle (7). Another approach is to dissect the suspected non-RLN in a retrograde manner, starting from its insertion into the cricothyroid muscle, keeping track of it in the neck.

The results of our study are in accordance with the findings of Henry et al. In their meta-analysis, the prevalence of an ARSA 
coexisting with a right non-RLN was $89.3 \%$, after reviewing 136 cases of right non-RLN. Furthermore, 2 cases of left non-RLNs were associated with situs inversus (17).

Even though the prevalence of non-RLN is estimated to range between $0.3-0.8 \%$ and $0.004 \%$ for right and left non-RLN respectively, cadaveric studies suggest a higher prevalence of $2.2 \%(3,11)$. In addition, intraoperative neuromonitoring may further increase those numbers up to $6 \%$ (7). Those discrepancies in prevalence suggest that even though non-RLN is a rare anatomic entity, it is rarely reported or there is a failure to recognize it. Therefore, the importance of proper and accurate preoperative imaging combined with intraoperative neuromonitoring is emphasized in increasing the detection rate of non-RLN. Furthermore, all cases of non- RLN and other rare anatomic variants need to be reported in nationwide registries.

\section{Conclusions}

Recurrent laryngeal nerve palsy is one of the complications of thyroid surgery with a reported incidence of 1-2\%. A non-RLN may be a rare anatomic variant with an incident reaching $0.3-2.2 \%$, but prediction and recognition of its presence can save the surgeon and the patient from potential injury to it, resulting in devastating results. This review aims to highlight the high degree of caution the surgeon must maintain when faced with anatomic variants of the great vessels, regarding the presence of a non-RLN. As shown in our review, the ARSA is almost always accompanied by a right non-RLN, with an incidence of $97 \%$. In the case of the extremely rare left non-RLN, situs inversus or a right aortic arch is observed. Preoperative identification of vascular variants suggests the presence of a non- recurrent laryngeal nerve. Further research is needed to recognize specific anatomical landmarks for its preoperative and intraopera- tive identification. The role of preoperative imaging, such as ultrasound, computerized tomography and magnetic resonance imaging, needs to be thoroughly evaluated. Neck ultrasound is a readily available and inexpensive method to detect vascular variations related to a non-RLN as an adjunct to thyroid ultrasound. However, the results are operator dependent. CT and MRI scans may give more detailed anatomical information but have the disadvantage of higher cost as well as a high dose of radiation in the case of a CT scan. Careful dissection with the aim of identifying the RLN is imperative. Constant intraoperative neuro-monitoring helps the surgeon identify and preserve the recurrent laryngeal nerve, and is associated with a higher identification rate of non-RLN at nearly $6 \%$. Although rare, the non-RLN in the hands of an unprepared surgeon may become their surgical Waterloo. It is a surgeon's responsibility to recognize the presence and preserve the anatomic integrity of such a rare neural entity. Further research is warranted for the identification of specific anatomic landmarks pointing to a non-RLN and the adoption of efficient preoperative and intraoperative methods to identify this elusive anatomic entity.

What is already known on this topic

Non-RLN is a rare anatomic variant of the inferior laryngeal nerve. Although rare, knowledge of its presence is fundamental in order to avoid injury to it during thyroid surgery.

\section{What this study adds}

This study aims to highlight the relationship between non-RLN and vascular variants. When a patient is shown to have anatomic variants of the great vessels, the surgeon should be aware of the presence of a non-RLN and opt to preserve it. As shown in our study, an ARSA is associated with a right non-RLN in 97\% of cases, while the extremely rare left non-RLN is usually associated with a right aortic arch.

Author's contributions: Conception and design: EB; Acquisition, analysis and interpretation of data: EB, IM, TD and GT; Drafting the article: EB and IM; Figure design: EB; Revising it critically for important intellectual content: EB, PS and DF; Approved final version of the manuscript: EB, IM, TD, GT, PS and DF. 
Conflict of interest: The authors declare that they have no conflict of interest.

\section{References}

1. Sosa JA, Hanna JW, Robinson KA, Lanman RB. Increases in Thyroid Nodule Fine-Needle Aspirations, Operations, and Diagnoses of Thyroid Cancer in the United States. Surgery. 2013;154(6):1420-6.

2. Becker WF. Presidential address: Pioneers in thyroid surgery. Ann Surg. 1977;185(5):493-504.

3. Mahmodlou R, Aghasi MR, Sepehrvand N. Identifying the non-recurrent laryngeal nerve: preventing a major risk of morbidity during thyroidectomy. Int J Prev Med. 2013;4(2):237-40.

4. Toniato A, Mazzarotto R, Piotto A, Bernante P, Pagetta C, Pelizzo MR. Identification of the nonrecurrent laryngeal nerve during thyroid surgery: 20-year experience. World J Surg. 2004;28(7):65961.

5. Pemberton J, Beaver MG. Anomaly of the right recurrent laryngeal nerve. Surg Gynecol Obstet. 1932;54:594-5.

6. Stedman G. A singular distribution of some of the nerves and arteries of the neck and the top of the thorax. Edinb Med Surg J. 1823;19(77):564-5.

7. Donatini G, Carnaille B, Dionigi G. Increased detection of non-recurrent inferior laryngeal nerve (NRLN) during thyroid surgery using systematic intraoperative neuromonitoring (IONM). World J Surg. 2013;37(1):91-3.

8. Guerreiro S, Lamas M, Candeias H, Eusebio R, Vitor Rocha. The non-recurrent laryngeal nerve: An anatomical "trap". Rev Port Endocrinol Diabetes Metab. 2014;9(1):84-7.

9. Morais M, Capela-Costa J, Matos-Lima L, CostaMaia J. Nonrecurrent Laryngeal Nerve and Associated Anatomical Variations: The Art of Prediction. Eur Thyroid J. 2015;4(4):234-8.

10. Uludag M, Isgor A, Yetkin G, Gitgez B. Anatomic variations of the non-recurrent inferior laryngeal nerve. BMJ Case Reports. 2009;2009: bcr10.2008.1107.

11. Hong KH, Park HT, Yang YS. Characteristic traveling patterns of non-recurrent laryngeal nerves. J Laryngol Otol. 2014;128(6):534-9.

12. Natsis K, Didagelos M, Gkioulava A, Lazaridis N, Vyzas V, Piagkou M. The aberrant right subclavian artery: cadaveric study and literature review. Surg
Radiol Anat. 2017;39(5):559-65. Erratum in: Surg Radiol Anat. 2017;39(10):1181-2.

13. Baker A, Cottrill EE, Munizza O, McGillen K, McKinnon T, Goldenberg D. Intrathyroidal carotid artery and nonrecurring right recurrent laryngeal nerve: A case report. Head Neck. 2017;39(12):123-6.

14. Obaid T, Kulkarni N, Pezzi TA, Turkeltaub AE, Pezzi CM. Coexisting right nonrecurrent and right nonrecurrent inferior laryngeal nerves: a rare and controversial entity; Report of a case and review of literature. Surg Today 2014;44(12):2392-6.

15. Sagayaraj A, Deo RP, Merchant S, Mohiyuddin SM, Nayak AC. Medially placed vagus nerve in relation to common carotid artery: a pointer to a non-recurrent laryngeal nerve. Eur Arch Otorhinolaryngol. 2015;272(10):3027-30.

16. Masuoka H, Miyauchi A, Higashiyama T, Yabuta T, Kihara M, Miya A. Right-sided aortic arch and aberrant left subclavian artery with or without a left nonrecurrent inferior laryngeal nerve. Head Neck. 2016;38(10):2508-11.

17. Henry BM, Sanna S, Graves MJ, Vikse J, Sanna B, Tomaszewska IM, et al. The Non-Recurrent Laryngeal Nerve: a meta-analysis and clinical considerations. PeerJ. 2017;5:e3012.

18. Niu ZX, Zhang H, Chen LQ, Shi H, Peng J, Su LW, et al. Preoperative computed tomography diagnosis of non-recurrent laryngeal nerve in patients with esophageal carcinoma. Thorac Cancer. 2017;8(1):46-50.

19. Tateda M, Hasegawa J, Sagai S, Nakanome A, Katagiri K, Ishida E, et al. Nonrecurrent inferior laryngeal nerve without vascular anomaly as a genuine entity. Tohoku J Exp Med. 2008;216(2):133-7.

20. Henry JF, Audiffret J, Denizot A, Plan M. The nonrecurrent inferior laryngeal nerve. Review of 33 cases, including two on the left side. Surgery. 1988;104(6):977-84.

21. Marchesi M, Biffoni M, Faloci C, Nobili Benedetti R, Notari P, Mariotti F, et al. The inferior nonrecurrent laryngeal nerve: A report of 7 cases observed since 1987. G Chir. 2000;21(1-2):25-8.

22. Patron V, Thenint MA, Robard L, Boittin F, Babin E, Hitier M. A freak of nature. Eur Ann Otorhinolaryngol Head Neck Dis. 2013;130(6):355-7.

23. Iorgulescu R, Bistriceanu I, Badanoiu D, Calin C, Capatana C, Iordache N. Non-recurrent inferior laryngeal nerve: case report and review of the literature. J Med Life. 2014;7(4):90-4. 